\title{
CURVATURE BLOW UP IN BIANCHI VIII AND IX VACUUM SPACETIMES
}

\author{
HANS RINGSTRÖM
}

\begin{abstract}
The maximal globally hyperbolic development of non-Taub-NUT Bianchi IX vacuum initial data and of non-NUT Bianchi VIII vacuum initial data is $C^{2}$-inextendible. Furthermore, a curvature invariant is unbounded in the incomplete directions of inextendible causal geodesics.
\end{abstract}

\section{INTRODUCTION}

According to a conjecture by Belinskii, Khalatnikov and Lifshitz, the Bianchi IX spacetimes are a good model for the local behaviour of generic gravitational collapse, see Berger et al (1998) and references therein. Although they have been analyzed numerically, few statements concerning these spacetimes have been proven.

There are several formulations of Einstein's equations for Bianchi IX models. One is the Hamiltonian description due to Misner and another is the formulation by Wainwright and Hsu. A brief explanation of the different variables can be found in Hobill et al (1994). We will use the formulation developed by Wainwright and Hsu (1989). In section 2 we explain how these variables are obtained.

In order to formulate the main result of this paper we need to define what is meant by the different types of initial data. Let $G$ be a 3-dimensional Lie group, $e_{i}$, $i=1,2,3$ be a basis of the Lie algebra with structure constants determined by $\left[e_{i}, e_{j}\right]=\gamma_{i j}^{k} e_{k}$. If $\gamma_{i k}^{k}=0$, then the Lie algebra and Lie group are said to be of class A and

$$
\gamma_{i j}^{k}=\epsilon_{i j m} n^{k m}
$$

where the symmetric matrix $n^{i j}$ is given by

$$
n^{i j}=\frac{1}{2} \gamma_{k l}^{(i} \epsilon^{j) k l}
$$

Definition 1. Class A vacuum initial data for Einstein's equations consist of the following. A Lie group $G$ of class A, a left invariant metric $g$ on $G$ and a left invariant symmetric covariant two-tensor $k$ on $G$ satisfying

$$
R_{g}-k_{i j} k^{i j}+\left(\operatorname{tr}_{g} k\right)^{2}=0
$$

and

$$
\nabla_{i} \operatorname{tr}_{g} k-\nabla^{j} k_{i j}=0
$$

where $\nabla$ is the Levi-Civita connection of $g$ and $R_{g}$ is the corresponding scalar curvature, indices are raised and lowered by $\mathrm{g}$. 
TABle 1. Bianchi class A.

$\begin{array}{lccc}\text { Type } & n_{1} & n_{2} & n_{3} \\ \text { I } & 0 & 0 & 0 \\ \text { II } & + & 0 & 0 \\ \text { VI }_{0} & 0 & + & - \\ \text { VII }_{0} & 0 & + & + \\ \text { VIII } & - & + & + \\ \text { IX } & + & + & +\end{array}$

We can choose a left invariant orthonormal basis $\left\{e_{i}\right\}$ with respect to $g$ so that the corresponding matrix $n^{i j}$ defined in (2) is diagonal with diagonal elements $n_{1}, n_{2}$ and $n_{3}$. By an appropriate choice of orthonormal basis $n_{1}, n_{2}, n_{3}$ can be assumed to belong to one and only one of the types given in table 1. We assign a Bianchi type to the initial data accordingly. This division constitutes a classification of the class A Lie algebras. We refer to Lemma 1 for a proof of these statements.

Let $k_{i j}=k\left(e_{i}, e_{j}\right)$. Then the matrices $n^{i j}$ and $k_{i j}$ commute according to (4i) so that we may assume $k_{i j}$ to be diagonal with diagonal elements $k_{1}, k_{2}$ and $k_{3}$, cf. (6).

Definition 2. Class A vacuum initial data, except type I and VII $I_{0}$ data with $\operatorname{tr}_{g} k=$ 0 , satisfying $k_{2}=k_{3}$ and $n_{2}=n_{3}$ or one of the permuted conditions are said to be non-generic. In the Bianchi IX and VIII cases we call such data Taub-NUT and NUT vacuum initial data respectively.

We will justify this definition in the remark following Lemma 2. Observe that the condition is independent of the choice of orthonormal basis diagonalizing $n$ and $k$, cf. (6). What is meant by inextendibility is explained in the following

Definition 3. Consider a connected Lorentz manifold $(M, g)$. If there is a connected $C^{2}$ Lorentz manifold $(\hat{M}, \hat{g})$ of the same dimension and a map $i: M \rightarrow \hat{M}$, with $i(M) \neq \hat{M}$, which is an isometry onto its image, then $(M, g)$ is said to be $C^{2}$-extendible and $(\hat{M}, \hat{g})$ is called a $C^{2}$-extension of $(M, g)$. A Lorentz manifold which is not $C^{2}$-extendible is said to be $C^{2}$-inextendible.

Remark. There is an analogous definition of smooth extensions. Unless otherwise mentioned manifolds are assumed to be smooth and maps between manifolds are assumed to be as regular as possible.

We are now in a position to state the main theorem.

Theorem 1. Consider the maximal globally hyperbolic development of non-TaubNUT Bianchi IX vacuum initial data or of non-NUT Bianchi VIII vacuum initial data. It is $C^{2}$-inextendible and the Kretschmann scalar

$$
\kappa=R_{\alpha \beta \gamma \delta} R^{\alpha \beta \gamma \delta}
$$

is unbounded in the incomplete directions of inextendible causal geodesics.

Remark. One can time orient the Bianchi VIII development mentioned in Theorem 11 so that all causal geodesics are future complete and past incomplete. In the Bianchi IX development mentioned, all causal geodesics are future and past incomplete. A proof, not the first, is given in Lemma 2. 
We also obtain qualitative information concerning the asymptotic behaviour of solutions in the causally geodesically incomplete directions. A solution which is not NUT nor Taub-NUT must oscillate indefinitely in the variables of Wainwright and Hsu as one approaches a singularity.

In his article concerning the global dynamics of the Mixmaster model, Rendall (1997) proved, among other things, the corresponding theorem for Bianchi type I (with $\left.\operatorname{tr}_{g} k \neq 0\right), \mathrm{II}, \mathrm{VI}_{0}$ and $\mathrm{VII}_{0}\left(\operatorname{tr}_{g} k \neq 0\right)$ vacuum initial data, the exceptional cases being the non-generic data. Bianchi type $\mathrm{I}$ and $\mathrm{VII}_{0}$ vacuum initial data satisfying $\operatorname{tr}_{g} k=0$ yield geodesically complete, and consequently inextendible, maximal globally hyperbolic developments, see Lemma 2. Thus we have

Corollary 1. Strong Cosmic Censorship holds for Bianchi class A vacuum initial data. That is, the maximal globally hyperbolic development of generic vacuum initial data of class $A$ is $C^{2}$-inextendible.

For a related paper see Chruściel and Rendall (1995). They isolate the locally homogeneous vacuum initial data on compact manifolds whose maximal globally hyperbolic developments have smooth extensions. In the Bianchi IX case, they prove that the homogeneous vacuum initial data that lead to an extendible maximal globally hyperbolic development are the Taub-NUT initial data. They also show, in the compact, locally homogeneous case, that extendibility implies additional symmetry of the solution in the sense that the local Killing algebra has dimension at least four. Thus they are able to conclude that extendibility is non-generic.

Our arguments can be extended to a more general situation. Consider initial data as in Definition 1 with $G$ simply connected. If $\Gamma$ is a subgroup of $G$ that acts freely and properly discontinuously on $G$ on the left, then we get well defined initial data on the quotient $G / \Gamma$ and our results apply to them. However, one does not necessarily obtain all quotients of $G$ that admit locally homogeneous data in this way. One can for instance take quotients of $\mathbb{R}^{3}$ by a subgroup of the isometry group (of the standard flat metric) which is not a subgroup of the group of translations. In certain situations the process of taking the quotient yields restrictions on the possible initial data. These restrictions have to be analyzed for one to be able to answer questions concerning cosmic censorship. We refer to Chruściel and Rendall (1995) for further elaboration on this point.

Section 2 contains a sketch of a derivation of the evolution equations. The first form of the equations presented there is due to Ellis and MacCallum (1969). We then reformulate them as in the article by Wainwright and Hsu (1989) and state some curvature expressions we will need later. The remaining sections contain the proof.

\section{The equations of Wainwright and Hsu}

In this section we consider a special class of spatially homogeneous four dimensional vacuum spacetimes of the form

$$
\left(I \times G,-d t^{2}+\chi_{i j}(t) \xi^{i} \otimes \xi^{j}\right)
$$

where $I$ is an open interval, $G$ is a Lie group of class A, $\chi_{i j}$ is a smooth positive definite matrix and the $\xi^{i}$ are the duals of a left invariant basis on $G$. Below, Latin indices will be raised and lowered by $\delta_{i j}$. 
Lemma 1. Table 1 constitutes a classification of the class A Lie algebras. Consider an arbitrary basis $\left\{e_{i}\right\}$ of the Lie algebra. Then by applying an orthogonal matrix to it, we can construct a basis $\left\{e_{i}^{\prime}\right\}$ such that the corresponding $n^{\prime}$ defined by (G) has diagonal elements of one of the types given in table 1 .

Proof. Let $e_{i}$ be a basis for the Lie algebra and $n$ be defined as in (2). If we change the basis according to $e_{i}^{\prime}=\left(A^{-1}\right)_{i}{ }^{j} e_{j}$ then $n$ transforms to

$$
n^{\prime}=(\operatorname{det} A)^{-1} A^{t} n A
$$

Since $n$ is symmetric we assume from here on that the basis is such that it is diagonal. The matrix $A=\operatorname{diag}\left(\begin{array}{lll}1 & 1 & -1\end{array}\right)$ changes the sign of $n$. A suitable orthogonal matrix performs even permutations of the diagonal. The number of non-zero elements on the diagonal is invariant under transformations (6) taking one diagonal matrix to another. If $A=\left(a_{i j}\right)$ and the diagonal matrix $n^{\prime}$ is constructed as in (6) we have $n_{k k}^{\prime}=(\operatorname{det} A)^{-1} \sum_{i=1}^{3} a_{i k}^{2} n_{i i}$ so that if all the diagonal elements of $n$ have the same sign the same is true for $n^{\prime}$. The statements of the lemma follow.

Let $(M, g)=\left(I \times G,-d t^{2}+\chi_{i j}(t) \xi^{i} \otimes \xi^{j}\right)$ be four dimensional and $G$ be of class A. Let $e_{0}=\partial_{t}$ and $e_{i}=a_{i}{ }^{j} Z_{j}, \mathrm{i}=1,2,3$, be an orthonormal basis, where $a$ is a $C^{\infty}$ matrix valued function of $t$ and the $Z_{i}$ are the duals of $\xi^{i}$. Let the matrix $A$ satisfy $e_{0}(A)+A B=0, A(0)=\mathrm{Id}$ where $B_{i j}=<\nabla_{e_{0}} e_{i}, e_{j}>$ and Id is the $3 \times 3$ identity matrix. Then $A$ is smooth and $S O(3)$ valued and if $e_{i}^{\prime}=A_{i}{ }^{j} e_{j}$ then $<\nabla_{e_{0}} e_{i}^{\prime}, e_{j}^{\prime}>=0$. Assume $<\nabla_{e_{0}} e_{i}, e_{j}>=0$. Observe that $\left[Z_{i}, e_{0}\right]=0$. The $e_{i}$ span the tangent space of $G$ and $<\left[e_{0}, e_{i}\right], e_{0}>=0$. Let

$$
\theta(X, Y)=<\nabla_{X} e_{0}, Y>
$$

$\theta_{\alpha \beta}=\theta\left(e_{\alpha}, e_{\beta}\right)$ and $\left[e_{\beta}, e_{\gamma}\right]=\gamma_{\beta \gamma}^{\alpha} e_{\alpha}$ where Greek indices run from 0 to 3 . The objects $\theta_{\alpha \beta}$ and $\gamma_{\beta \gamma}^{\alpha}$ will be viewed as smooth functions from $I$ to some suitable $\mathbb{R}^{k}$. We get $\theta_{00}=\theta_{0 i}=0$ and $\theta_{\alpha \beta}$ symmetric. We also have $\gamma_{i j}^{0}=\gamma_{0 i}^{0}=0$ and $\gamma_{0 j}^{i}=-\theta_{i j}$. We let $n$ be defined as in (2) and

$$
\sigma_{i j}=\theta_{i j}-\frac{1}{3} \theta \delta_{i j}
$$

where we by abuse of notation have written $\operatorname{tr}(\theta)$ as $\theta$.

We compute the Einstein tensor in terms of $n, \sigma$ and $\theta$. The Jacobi identities for $e_{\alpha}$ yield

$$
e_{0}\left(n_{i j}\right)-2 n_{k(i} \sigma_{j)}^{k}+\frac{1}{3} \theta n_{i j}=0 .
$$

The $0 i$-components of the Einstein equations are

$$
\sigma_{i}{ }^{k} n_{k j}-n_{i}{ }^{k} \sigma_{k j}=0 .
$$

Letting $b_{i j}=2 n_{i}{ }^{k} n_{k j}-\operatorname{tr}(n) n_{i j}$ and $s_{i j}=b_{i j}-\frac{1}{3} \operatorname{tr}(b) \delta_{i j}$ the trace free part of the $i j$ equations are

$$
e_{0}\left(\sigma_{i j}\right)+\theta \sigma_{i j}+s_{i j}=0
$$

The fact that $R_{00}=0$ yields the Raychaudhuri equation

$$
e_{0}(\theta)+\theta_{i j} \theta^{i j}=0
$$


and using this together with the trace of the $i j$-equations yields a constraint

$$
\sigma_{i j} \sigma^{i j}+\left(n_{i j} n^{i j}-\frac{1}{2} \operatorname{tr}(n)^{2}\right)=\frac{2}{3} \theta^{2}
$$

Equations (8)-(12) are special cases of equations given in Ellis and MacCallum (1969). At a point $t_{0}$ we may diagonalize $n$ and $\sigma$ simultaneously since they commute (9). Rotating $e_{\alpha}$ by the corresponding element of $S O(3)$ yields upon going through the definitions that the new $n$ and $\sigma$ are diagonal at $t_{0}$. Collect the offdiagonal terms of $n$ and $\sigma$ in one vector $v$. By (8) and (10) there is a time dependent matrix $C$ such that $\dot{v}=C v$ so that $v(t)=0 \forall t$ since $v\left(t_{0}\right)=0$. Since the rotation was time independent $\left\langle\nabla_{e_{0}} e_{i}, e_{j}\right\rangle=0$ holds in the new basis.

In order to prove curvature blow up, we need to relate the maximal existence interval for solutions to (8)-(12) to the maximal globally hyperbolic development. One ingredient is the following lemma, the proof of which can be found in the appendix.

Lemma 2. Let $(G, g, k)$ be class $A$ vacuum initial data. We can then choose a left invariant orthonormal basis $\left\{e_{i}^{\prime}\right\}$ with respect to $g$ so that the corresponding $n^{\prime}$ given by (国) is of one of the types given in table 1 . We can also assume $k_{i j}=k\left(e_{i}^{\prime}, e_{j}^{\prime}\right)$ to be diagonal. Then there is a manifold as in (5) where $I=\left(t_{-}, t_{+}\right)$is the maximal existence interval for a solution of (\$) - (12) with initial data $n\left(t_{0}\right)=n^{\prime}, \sigma_{i j}\left(t_{0}\right)=$ $k_{i j}-\operatorname{tr}(k) \delta_{i j} / 3$ and $\theta\left(t_{0}\right)=\operatorname{tr}(k)$, solving Einstein's vacuum equations, such that the metric restricted to $M_{t_{0}}=\left\{t_{0}\right\} \times G$ is $g$ and $k$ is the second fundamental form of $M_{t_{0}}$. The development has the following properties:

1. Each $M_{v}=\{v\} \times G, v \in I$, is a Cauchy surface.

2. If the initial data is not of type IX, the development can be time oriented so that it is future causally geodesically complete and past causally geodesically incomplete (unless the data is of type I or VII $I_{0}$ with $\operatorname{tr}_{g} k=0$ in which case the development is causally geodesically complete). We assume this time orientation when we speak of such developments, and always that $\partial_{t}$ is future oriented. The development of Bianchi IX initial data is both future and past causally geodesically incomplete.

3. The causally geodesically complete developments have $\theta=0$ and $\sigma_{i j}=0$ for the entire solution. The developments with one complete and one incomplete direction have $\theta>0$ for the entire solution. Bianchi IX developments have $\theta>0$ in $\left(t_{-}, t_{0}\right)$ and $\theta<0$ in $\left(t_{0}, t_{+}\right)$for some $t_{0} \in\left(t_{-}, t_{+}\right)$.

4. If the Kretschmann scalar $\kappa$ is unbounded as $t \rightarrow t_{-}$then it is unbounded along past inextendible causal geodesics. Similarly for $t \rightarrow t_{+}$and future oriented causal geodesics.

Remark. By inspecting (8) and (10) we see that if $k_{2}=k_{3}$ and $n_{2}^{\prime}=n_{3}^{\prime}$ then $\sigma_{2}=\sigma_{3}$ and $n_{2}=n_{3}$ for the entire solution. Since such solutions are of NUT and Taub-NUT type for the Bianchi VIII and IX cases respectively, see Ellis and MacCallum (1969), NUT and Taub-NUT vacuum initial data yield NUT and Taub-NUT developments respectively. 
Introduce, as in Wainwright and Hsu (1989),

$$
\begin{aligned}
\Sigma_{i j} & =\sigma_{i j} / \theta \\
N_{i j} & =n_{i j} / \theta \\
B_{i j} & =2 N_{i}{ }^{k} N_{k j}-N_{k}^{k} N_{i j} \\
S_{i j} & =B_{i j}-\frac{1}{3} B_{k}^{k} \delta_{i j}
\end{aligned}
$$

and define a new time coordinate $\tau$, independent of time orientation, by

$$
\frac{d t}{d \tau}=\frac{3}{\theta} .
$$

For Bianchi IX developments as in Lemma we only consider the part of spacetime where $\theta$ is strictly positive or strictly negative. Let $\Sigma_{+}=\frac{3}{2}\left(\Sigma_{22}+\Sigma_{33}\right)$ and $\Sigma_{-}=$ $\sqrt{3}\left(\Sigma_{22}-\Sigma_{33}\right) / 2$. If we let $N_{i}$ be the diagonal elements of $N_{i j}$, equations (8) and (10) turn into

$$
\begin{aligned}
N_{1}^{\prime} & =\left(q-4 \Sigma_{+}\right) N_{1} \\
N_{2}^{\prime} & =\left(q+2 \Sigma_{+}+2 \sqrt{3} \Sigma_{-}\right) N_{2} \\
N_{3}^{\prime} & =\left(q+2 \Sigma_{+}-2 \sqrt{3} \Sigma_{-}\right) N_{3} \\
\Sigma_{+}^{\prime} & =-(2-q) \Sigma_{+}-3 S_{+} \\
\Sigma_{-}^{\prime} & =-(2-q) \Sigma_{-}-3 S_{-}
\end{aligned}
$$

where the prime denotes derivative with respect to $\tau$ and

$$
\begin{aligned}
q & =2\left(\Sigma_{+}^{2}+\Sigma_{-}^{2}\right) \\
S_{+} & =\frac{1}{2}\left[\left(N_{2}-N_{3}\right)^{2}-N_{1}\left(2 N_{1}-N_{2}-N_{3}\right)\right] \\
S_{-} & =\frac{\sqrt{3}}{2}\left(N_{3}-N_{2}\right)\left(N_{1}-N_{2}-N_{3}\right) .
\end{aligned}
$$

The vacuum constraint (12) becomes

$$
\Sigma_{+}^{2}+\Sigma_{-}^{2}+\frac{3}{4}\left[N_{1}^{2}+N_{2}^{2}+N_{3}^{2}-2\left(N_{1} N_{2}+N_{2} N_{3}+N_{3} N_{1}\right)\right]=1 .
$$

The equations (14)-(16) have certain symmetries described in Wainwright and Hsu (1989). By permuting $N_{1}, N_{2}, N_{3}$ arbitrarily we get new solutions if we at the same time carry out appropriate combinations of rotations by integer multiples of $2 \pi / 3$ and reflections in the $\left(\Sigma_{+}, \Sigma_{-}\right)$-plane. Below we refer to rotations by integer multiples of $2 \pi / 3$ as rotations. Changing the sign of all the $N_{i}$ at the same time does not change the equations. Classify points $\left(N_{1}, N_{2}, N_{3}, \Sigma_{+}, \Sigma_{-}\right)$according to the values of $N_{1}, N_{2}, N_{3}$ in the same way as in table 1. Since the sets $N_{i}>0$, $N_{i}<0$ and $N_{i}=0$ are invariant under the flow of the equation we may classify solutions to (14)-(16) accordingly. When we speak of Bianchi IX solutions we will assume $N_{i}>0$, and for Bianchi VIII solutions we will assume two $N_{i}>0$ and one $<0$.

There are three special points in the $\left(\Sigma_{+}, \Sigma_{-}\right)$-plane. They are $(-1,0)$ and $(1 / 2, \pm \sqrt{3} / 2)$ on the Kasner circle $q=2$. They are rotated into one another by applying the symmetries and the corresponding type I solutions represent isometric flat universes. If 
the spatial topology is $\mathbb{R}^{3}$, the metric of these solutions can be written as

$$
d s^{2}=-d t^{2}+\sum_{i=1}^{3} t^{2 p_{i}} d x^{i} \otimes d x^{i}
$$

on the manifold $M=\mathbb{R}_{+} \times \mathbb{R}^{3}$, where $\mathbb{R}^{+}=\{t \in \mathbb{R}: t>0\}$, with two of the $p_{i}$ zero and one equal to 1 . The rotational symmetries in the $\Sigma_{+} \Sigma_{-}$-plane correspond to permutational symmetry of the $p_{i}$. We see that if for instance $p_{2}=p_{3}=0$ we have a rotational symmetry in the $x^{2} x^{3}$-plane at each point of $M$.

The set $\Sigma_{-}=0, N_{2}=N_{3}$ is invariant under the flow of (14)-(16). Applying the symmetries to this set we get new invariant sets. These sets correspond to the nongeneric data in Definition 2. Specifically, in the Bianchi IX case they correspond to the Taub-NUT solutions.

The Raychaudhuri equation (11) takes the form

$$
\theta^{\prime}=-(1+q) \theta
$$

According to Lemma 5 the existence interval for solutions of (14)-(16) is past infinite. As $\tau \rightarrow-\infty, \theta(\tau)$ goes to infinity exponentially according to (18).

The normalized Kretschmann scalar is given by

$$
\tilde{\kappa}=R_{\alpha \beta \gamma \delta} R^{\alpha \beta \gamma \delta} / \theta^{4} \text {. }
$$

We have

$$
\tilde{\kappa}=8\left(E_{i j} E^{i j}-H_{i j} H^{i j}\right)
$$

where, by Wainwright and Ellis (1997), the normalized $E$ and $H$ fields are given by

$$
\begin{aligned}
& E_{i j}=\frac{1}{3} \Sigma_{i j}-\left(\Sigma_{i}{ }^{k} \Sigma_{k j}-\frac{2}{3} \Sigma^{2} \delta_{i j}\right)+S_{i j} \\
& H_{i j}=-3 \Sigma^{k}{ }_{i} N_{j) k}+N^{k l} \Sigma_{k l} \delta_{i j}+\frac{1}{2} N_{k}^{k} \Sigma_{i j}
\end{aligned}
$$

and $\Sigma^{2}=\Sigma_{i j} \Sigma^{i j} / 2$. We may consider all matrices involved to be 3 -vectors. If we write $E_{i j}$ as $E=\left(\begin{array}{lll}E_{1} & E_{2} & E_{3}\end{array}\right)$ and similarly for $H$ we have $\tilde{\kappa}=8\left(|E|^{2}-|H|^{2}\right)$. Using the definitions and the constraint (16) we compute

$$
\begin{aligned}
H_{1} & =N_{1} \Sigma_{+}+\frac{1}{\sqrt{3}}\left(N_{2}-N_{3}\right) \Sigma_{-} \\
H_{2} & =-\frac{1}{2} N_{2}\left(\Sigma_{+}+\sqrt{3} \Sigma_{-}\right)+\frac{1}{2}\left(N_{3}-N_{1}\right)\left(\Sigma_{+}-\frac{1}{\sqrt{3}} \Sigma_{-}\right) \\
E_{2}-E_{3} & =\frac{2}{3 \sqrt{3}} \Sigma_{-}\left(1-2 \Sigma_{+}\right)+\left(N_{2}-N_{3}\right)\left(N_{2}+N_{3}-N_{1}\right) \\
E_{2}+E_{3} & =\frac{2}{9} \Sigma_{+}+\frac{10}{9} \Sigma_{+}^{2}+\frac{2}{3} \Sigma_{-}^{2}-\frac{8}{9}+\left(N_{2}-N_{3}\right)^{2}-N_{1}\left(N_{2}+N_{3}\right)
\end{aligned}
$$

The fact that $E$ and $H$ are traceless yields the remaining components. We end this section with a technical lemma relating the existence intervals for the variables of Wainwright and Hsu and those of Ellis and MacCallum.

Lemma 3. If for all non-Taub-NUT Bianchi IX initial data for (14)-(10) we can prove the existence of a sequence $\tau_{k} \rightarrow-\infty$ such that $\tilde{\kappa}\left(\tau_{k}\right)$ does not converge to zero, then for every development as in Lemma Of non-Taub-NUT initial data, the Kretschmann scalar is unbounded as $t \rightarrow t_{-}$and $t \rightarrow t_{+}$(here $t_{+}$and $t_{-}$are the 



VIII initial data of (14)-(10) we can prove the existence of a sequence $\tau_{k} \rightarrow-\infty$ such that $\tilde{\kappa}\left(\tau_{k}\right)$ does not converge to zero, then for every development as in Lemma g of non-NUT initial data, the Kretschmann scalar is unbounded as $t \rightarrow t_{-}$.

Remark. That the existence interval for solutions to (14)-(16) is past infinite is proven in Lemma 5 .

\section{Elementary properties of SOlutions}

We begin by giving the past existence intervals for solutions to (14)-(16). Let $g \in C^{\infty}\left(\mathbb{R}^{n}, \mathbb{R}^{n}\right)$ and consider $\dot{x}=g(x), x(0)=x_{0}$. Let $\left(t_{-}, 0\right]$ be the maximal past existence interval for the solution.

Lemma 4. If $t_{-}>-\infty$ there is no sequence $t_{k} \rightarrow t_{-}, t_{k} \in\left(t_{-}, 0\right]$, such that $x\left(t_{k}\right)$ converges.

Proof. Assume $t_{k} \rightarrow t_{-}, x\left(t_{k}\right) \rightarrow x_{-}$. There is an $\epsilon>0$ and a $\delta>0$ such that we have a smooth flow

$$
\Phi: B_{\epsilon}\left(x_{-}\right) \times(-\delta, \delta) \rightarrow \mathbb{R}^{n}
$$

where $B_{\epsilon}\left(x_{-}\right)$is the open ball with radius $\epsilon$ and center $x_{-}$. Choose a $k$ such that $x\left(t_{k}\right) \in B_{\epsilon}\left(x_{-}\right)$and $\left|t_{k}-t_{-}\right|<\delta / 2$. Define $y(t)=x(t)$ for $t \in\left(t_{k}, 0\right]$ and $y\left(t_{k}-t\right)=\Phi\left(x\left(t_{k}\right), t\right)$ for $t \in[0, \delta)$. Then $y$ extends $x$ beyond the maximal existence interval.

Lemma 5. The past existence interval for solutions to (14)- 10 id $(-\infty, 0]$.

Proof. For all types except IX the constraint implies $\Sigma_{+}^{2}+\Sigma_{-}^{2} \leq 1$ so that $N_{1}, N_{2}, N_{3}$ do not grow faster than exponentially, by (14). Let $\left(\tau_{-}, \tau_{+}\right)$be the maximal existence interval for a specific solution, not of type IX. If $\tau_{-}>-\infty$ we may for any sequence $\tau_{k}>\tau_{-}$converging to $\tau_{-}$extract a subsequence such that the solution converges, contradicting Lemma 1 . In other words we may not have $\tau_{-}>-\infty$, and in the same way we have $\tau_{+}=\infty$. Consider solutions of type IX. Let $\tau \leq 0$. We get

$$
N_{1}(\tau)=\exp \left\{\int_{0}^{\tau}\left[q(s)-4 \Sigma_{+}(s)\right] d s\right\} N_{1}(0) \leq e^{-2 \tau} N_{1}(0)
$$

and similarly for $N_{2}$ and $N_{3}$. Thus, the $N_{i}$ do not grow faster than exponentially as we go backward in time and by the constraint the same holds for $\left(\Sigma_{+}, \Sigma_{-}\right)$. The past existence interval must be $(-\infty, 0]$.

We will need a few more lemmas concerning the behaviour of solutions of type VIII and IX.

Lemma 6. Consider a solution of type IX. The image $\left(\Sigma_{+}, \Sigma_{-}\right)((-\infty, 0])$ is contained in a compact set whose size depends on the initial data. Further, if at a point $N_{3} \geq N_{2} \geq N_{1}$ and $N_{3} \geq 2$, then $N_{2} \geq N_{3} / 10$.

Remark. The importance of the second part of the lemma is to be found in the consequence that one of the $N_{i}$ may not go to infinity alone. 
Proof. Let us prove the second statement first. Assume $N_{3} \geq 2$ and $N_{3} \geq 10 N_{2} \geq$ $10 N_{1}$, so that $-N_{2} \geq-N_{3} / 10$ and similarly for $N_{1}$. We get

$$
\left(N_{1}-N_{2}\right)^{2}+N_{3}^{2}-2 N_{3}\left(N_{1}+N_{2}\right) \geq \frac{12}{5}
$$

contradicting the constraint (16). We are done. Since $\left(N_{1} N_{2} N_{3}\right)^{\prime}=3 q N_{1} N_{2} N_{3}$ the product decreases as time decreases, and we have

$$
\left(N_{1} N_{2} N_{3}\right)(\tau) \leq C<\infty
$$

for $\tau \leq 0$, where $C$ is a constant. There is a compact set $K_{1}$ such that if $N_{i} \leq 2$, $i=1,2,3$, then $\left(\Sigma_{+}, \Sigma_{-}\right) \in K_{1}$. If one of the $N_{i}$ is greater than 2 , we may by a permutation assume that $N_{3} \geq N_{2} \geq N_{1}$ and by the first part of the lemma conclude that $N_{2} \geq N_{3} / 10$. Observe that then $N_{1}\left(N_{2}+N_{3}\right)<10 C$ so that

$$
\Sigma_{+}^{2}+\Sigma_{-}^{2} \leq 1+15 C
$$

by the constraint.

Lemma 7. Consider a solution of type VIII. Assume $N_{3} \geq N_{2}>0>N_{1}$. Then $N_{1}^{2} \leq 4 / 3$. If further $N_{3} \geq 4$ then $N_{2} \geq N_{3} / 2$.

Proof. The constraint says

$$
\Sigma_{+}^{2}+\Sigma_{-}^{2}+\frac{3}{4}\left[N_{1}^{2}+\left(N_{2}-N_{3}\right)^{2}-2 N_{1}\left(N_{2}+N_{3}\right)\right]=1 .
$$

Since all terms on the left are non-negative, the first part of the lemma is immediate. The second part follows from

$$
N_{3}-N_{2} \leq \frac{2}{\sqrt{3}} \leq 2 \leq \frac{1}{2} N_{3}
$$

We end this section with a lemma needed in the sequel. Consider a positive function $N$ satisfying $N^{\prime}=h N$ where $h(\tau) \rightarrow \alpha$ as $\tau \rightarrow-\infty$

Lemma 8. Assume $N$ and $h$ are as above. Then for all $\epsilon>0$ there is a $T$ such that $\tau \leq T$ implies

$$
\exp [(\alpha+\epsilon) \tau] \leq N(\tau) \leq \exp [(\alpha-\epsilon) \tau]
$$

\section{Limit CHARACTERIZATION OF SPECIAL SOLUtions}

Proposition 1. A solution to $(14)-(10)$ satisfies

$$
\lim _{\tau \rightarrow-\infty}\left(\Sigma_{+}(\tau), \Sigma_{-}(\tau)\right)=(-1,0)
$$

only if it is contained in the invariant set $\Sigma_{-}=0$ and $N_{2}=N_{3}$.

Remark. The implication is in fact an equivalence. The analogue for $\left(\Sigma_{+}, \Sigma_{-}\right) \rightarrow$ $(1 / 2, \pm \sqrt{3} / 2)$ is true due to the symmetries.

Assume in this section that $\left(\Sigma_{+}, \Sigma_{-}\right)$converges to $(-1,0)$. Consider

$$
f=\frac{4}{3} \Sigma_{-}^{2}+\left(N_{2}-N_{3}\right)^{2}
$$

Observe that $f$ is either identically zero or always strictly positive due to the fact that $N_{2}=N_{3}, \Sigma_{-}=0$ is an invariant set. A function related to this one occurs in 
Wainwright and Hsu (1989). We prove an estimate of the form $f(T) \leq g(\tau, T)$ for some $T$ and $\tau \leq T$ and then that $g(\tau, T) \rightarrow 0$ as $\tau \rightarrow-\infty$. Compute

$$
f^{\prime}=-(2-q) \frac{8}{3} \Sigma_{-}^{2}+4 \sqrt{3} N_{1}\left(N_{2}-N_{3}\right) \Sigma_{-}+2\left(q+2 \Sigma_{+}\right)\left(N_{2}-N_{3}\right)^{2} .
$$

Lemma 9. For all $\epsilon>0$ there is a $T$ such that

$$
f(T) \leq f(\tau) \exp [\epsilon(T-\tau)]
$$

for all $\tau \leq T$.

Proof. By Lemma $8 N_{1}$ goes to zero as $\tau \rightarrow-\infty$. The same is true of $2-q$ and $q+2 \Sigma_{+}$. Thus, for all $\epsilon>0$ there exists a $T$ such that $\tau \leq T$ implies $f^{\prime} \leq \epsilon f$ by (28). The lemma follows.

Lemma 10. If there is a sequence $\tau_{k} \rightarrow-\infty$ as $k \rightarrow \infty$ such that $\Sigma_{+}\left(\tau_{k}\right) \leq-1$, then $\Sigma_{-}=0$ and $N_{2}=N_{3}$.

Proof. The constraint yields, in the points $\tau_{k}$,

$$
\frac{3}{4} f=\Sigma_{-}^{2}+\frac{3}{4}\left(N_{2}-N_{3}\right)^{2} \leq \frac{3}{2} N_{1}\left(N_{2}+N_{3}\right) .
$$

Applying Lemma 8 to $N_{1} N_{2}$ and $N_{1} N_{3}$, we have, for $k$ large enough, $f\left(\tau_{k}\right) \leq$ $\exp \left(2 \tau_{k}\right)$. Choose a finite $T$ corresponding to $\epsilon=1$ in the previous lemma. We get $f(T) \leq \exp \left(\tau_{k}+T\right)$. Letting $k \rightarrow \infty$ we get $f(T)=0$, but then $f$ is identically zero.

Lemma 11. If there is an $S$ such that $\Sigma_{+}(\tau) \geq-1$ for all $\tau \leq S$ then $\Sigma_{-}=0$ and $N_{2}=N_{3}$.

Proof. Using the constraint to express $2-q$ in the $N_{i}$ we get, if we assume $-1 \leq$ $\Sigma_{+} \leq 0$

$$
\left(\Sigma_{+}+1\right)^{\prime} \leq 6 N_{1}^{2}+6\left|N_{1}\left(N_{2}+N_{3}\right)\right| .
$$

Let $T$ be such that the right hand side $\leq 2 e^{2 \tau}$ and $-1 \leq \Sigma_{+}(\tau) \leq 0$ for all $\tau \leq T$. Integrate the inequality to obtain

$$
0 \leq \Sigma_{+}(\tau)+1 \leq e^{2 \tau}
$$

for all $\tau \leq T$. But then the constraint yields

$$
\frac{3}{4} f=-\frac{3}{4} N_{1}^{2}+\frac{3}{2} N_{1}\left(N_{2}+N_{3}\right)+\left(1-\Sigma_{+}\right)\left(1+\Sigma_{+}\right) .
$$

By the above argument we have control over the last term, and as in the previous lemma we have control over the first two terms. Thus, there exists an $S^{\prime}$ such that $\tau \leq S^{\prime}$ implies $f(\tau) \leq e^{\tau}$. We deduce, using $\epsilon=\frac{1}{2}$ in Lemma 9 , that $f$ is identically zero.

\section{Properties of PASt Limit POINTS}

If there is a sequence $\tau_{k} \rightarrow-\infty$ such that a solution to (14)-(16) evaluated at $\tau_{k}$ converges to $x$, then $x$ is said to be an $\alpha$-limit point of the solution. The $\alpha$-limit set of a solution is the union of its $\alpha$-limit points. Consider a solution of type VIII or IX. Assume it has an $\alpha$-limit point $\left(n_{1}, n_{2}, n_{3}, s_{+}, s_{-}\right)$. The objective of this section is to prove that if $\left(s_{+}, s_{-}\right)$is not one of the special points it has an $\alpha$-limit point 
at which the normalized Kretschmann scalar is non-zero. The following lemma will be needed,

Lemma 12 (Rendall (1997)). Let $U$ be an open subset of $\mathbb{R}^{n}, f \in C^{\infty}\left(\mathbb{R}^{n}, \mathbb{R}^{n}\right)$ and let $F: U \rightarrow \mathbb{R}$ be a continuous function such that $F(x(t))$ is strictly monotone for any solution $x(t)$ of

$$
x^{\prime}(t)=f(x(t))
$$

as long as $x(t)$ is in $U$. Then no solution of (29) whose image is contained in $U$ has an $\alpha$-limit point in $U$.

As noted in Rendall (1997),

Lemma 13. If a solution of type VIII or IX has an $\alpha$-limit point $\left(n_{1}, n_{2}, n_{3}, s_{+}, s_{-}\right)$, then one of the $n_{i}$ must be zero.

Proof. We have

$$
\left|N_{1} N_{2} N_{3}\right|^{\prime}=3 q\left|N_{1} N_{2} N_{3}\right| \text {. }
$$

If $q=0$ then $\left|\Sigma_{+}^{\prime}\right|+\left|\Sigma_{-}^{\prime}\right|>0$ and consequently $\left|N_{1} N_{2} N_{3}\right|$ is strictly decreasing as we go backward in time if it is non-zero. The statement follows by Lemma 12.

Corollary 2. If a solution of type VIII or IX has an $\alpha$-limit point $\left(n_{1}, n_{2}, n_{3}, s_{+}, s_{-}\right)$, then $s_{+}^{2}+s_{-}^{2} \leq 1$.

Observe that points of type $\mathrm{VII}_{0}$ defined by $\Sigma_{+}=-1, \Sigma_{-}=0, N_{2}=N_{3} \neq 0$ and $N_{1}=0$ are fixed points to (14)-(16). The same is true of the rotated points. These points are called flat points of type $\mathrm{VII}_{0}$. These solutions correspond to the special points in the following sense. The vacuum spacetime given by the manifold $M=\mathbb{R}_{+} \times \mathbb{R}^{3}$ with a metric as in $(17)$ where $p_{1}=1$ and $p_{2}=p_{3}=0$ can be viewed as a type $\mathrm{VII}_{0}$ spacetime by giving $\mathbb{R}^{3}$ a different group structure. Then the Wainwright and Hsu variables corresponding to a suitable frame (left invariant under the new operation on $\mathbb{R}^{3}$ ) will be a flat point of type $\mathrm{VII}_{0}$.

Theorem 2 (Rendall (1997)). The $\alpha$-limit set of a solution of (14)-(16) of type I, II, VII or $V I I_{0}$ is a single point of type I or a flat point of type $V I I_{0}$, the latter only being possible if the solution is time independent.

Lemma 14. Consider a solution to (14)-(10) of type VIII or IX. If it has an $\alpha$ limit point $\left(n_{1}, n_{2}, n_{3}, s_{+}, s_{-}\right)$such that $\left(s_{+}, s_{-}\right)$is not special, it has at least two $\alpha$-limit points on the Kasner circle at least one of which is non-flat. In consequence there is an $\alpha$-limit point such that the normalized Kretschmann scalar evaluated at it is non-zero.

Proof. The solution of (14)-(16) with initial value $\left(n_{1}, n_{2}, n_{3}, s_{+}, s_{-}\right)$consists of $\alpha$-limit points to the original solution. Let us denote it $\left(N_{1}, N_{2}, N_{3}, \Sigma_{+}, \Sigma_{-}\right)$. The different solutions will be referred to as the solution and the original solution. Let us first prove that there is a non-flat $\alpha$-limit point on the Kasner circle. Since $\left(s_{+}, s_{-}\right)$ is not special we know that the solution is not a flat point of type $\mathrm{VII}_{0}$ and we can consequently assume that the $\alpha$-limit set of the solution is a single point of type I by Theorem 2, say $\left(0,0,0, \sigma_{+}, \sigma_{-}\right)$. If $\left(\sigma_{+}, \sigma_{-}\right)$is not special we are done so assume it is $(-1,0)$. By arguments given in the proof of Theorem 2, $N_{1}, N_{2}, N_{3}$ have to 
belong to a compact set as we go backward in time. If $\left(\Sigma_{+}, \Sigma_{-}\right)$does not converge to $(-1,0)$ we may thus construct an $\alpha$-limit point to the solution different from the only one that exists. By Proposition 1 we conclude that $\Sigma_{-}=0$ and $N_{2}=N_{3}$. If $N_{1}=0$ we get $\Sigma_{+}= \pm 1$ by the constraint so that $\Sigma_{+}=-1$ contradicting the fact that $\left(s_{+}, s_{-}\right)$is not special. Thus $N_{1} \neq 0$ and $N_{2}=N_{3}=0$. Applying the flow to this point we conclude that $\lim _{\tau \rightarrow \infty}\left(\Sigma_{+}(\tau), \Sigma_{-}(\tau)\right)=(1,0)$, again the details are to be found in the proof of Theorem 2. Since the $\alpha$-limit set is closed we conclude that the original solution has a non-flat $\alpha$-limit point on the Kasner circle.

We now prove that there are at least two $\alpha$-limit points on the Kasner circle. Given the non-flat limit point $\left(\sigma_{+}, \sigma_{-}\right)$, there is a neighbourhood $B_{\epsilon}\left(\sigma_{+}, \sigma_{-}\right)$of it in the $\Sigma_{+} \Sigma_{-}$-plane such that two $N_{i}$ decrease exponentially as $\exp (\alpha \tau)$ and one increases as $\exp (-\alpha \tau)$ for some $\alpha>0$ if $\left(\Sigma_{+}, \Sigma_{-}\right)$is in that neighbourhood. The solution must thus leave the neighbourhood as we go backward in time since otherwise Lemma 6 or Lemma 7 would yield a contradiction. If the solution evaluated at $\tau_{k}$ converges to the non-flat limit point on the Kasner circle, we can thus construct a sequence $s_{k}$ such that the $\Sigma_{+} \Sigma_{-}$-variables of the solution evaluated at it converges to a point on the boundary of $B_{\epsilon}\left(\sigma_{+}, \sigma_{-}\right)$. We can also assume that the $N_{i}$-variables of the solution, evaluated at $s_{k}$, converge. The limit point will have at most one $N_{i}$-variable non-zero by the construction. If all the $N_{i}$-variables are zero we have obtained a second $\alpha$-limit point on the Kasner circle. If one is non-zero, we may apply the flow to it to obtain two $\alpha$-limit points on the Kasner circle, cf. the proof of Theorem 2. Since the normalized Kretschmann scalar is non-zero on a non-flat limit point on the Kasner circle, see (22), the last statement follows.

\section{Existence of Limit POINTS}

In order to prove that an $\alpha$-limit point always exists we need a few lemmas. Let $\|N\|$ denote the Euclidean norm of $N=\left(N_{1}, N_{2}, N_{3}\right)$.

Lemma 15. Consider a Bianchi VIII or IX solution. If $\|N\| \rightarrow \infty$ as $\tau \rightarrow-\infty$ we can, by applying the symmetries to the solution, assume that $N_{2}, N_{3} \rightarrow \infty$ and $N_{1}, N_{1}\left(N_{2}+N_{3}\right) \rightarrow 0$ as $\tau \rightarrow-\infty$.

Proof. We have

$$
\left|N_{1} N_{2} N_{3}\right| \leq C \quad \forall \tau \leq 0
$$

where $C \geq 1$. Let $\tau_{0} \leq 0$ be such that

$$
N_{1}^{2}+N_{2}^{2}+N_{3}^{2} \geq 300 C^{2 / 3} \quad \forall \tau \leq \tau_{0} .
$$

If for $\tau \leq \tau_{0}\left|N_{1}\right|=\left|N_{2}\right| \leq\left|N_{3}\right|$ we get $N_{3} \geq 10 C^{1 / 3}$ and $\left|N_{1}\right|=\left|N_{2}\right| \geq C^{1 / 3}$ by inequality (31) and Lemma 6 or 7 . Since this is not reconcilable with inequality (30) we conclude that one $N_{i}$, say $N_{1}$, must satisfy $\left|N_{1}\right|<N_{2}$ and $\left|N_{1}\right|<N_{3} \forall \tau \leq \tau_{0}$. For a Bianchi VIII solution $N_{1}<0$. But then $N_{2}^{2}+N_{3}^{2} \leq 101 N_{2}^{2}$ by Lemma 6 or 8 and similarly for $N_{3}$. Consequently $N_{2}, N_{3} \rightarrow \infty$ as $\tau \rightarrow-\infty$. The rest of the conclusions of the lemma follow by inequality (30).

Lemma 16. Consider a Bianchi IX solution. If $-1<\Sigma_{+}\left(\tau_{1}\right)<1 / 3$ and $9 N_{1}<$ $N_{2}+N_{3}$ in $\left[\tau_{2}, \tau_{1}\right]$ then $\Sigma_{+}\left(\tau_{1}\right) \leq \Sigma_{+}(\tau)$ for $\tau \in\left[\tau_{2}, \tau_{1}\right]$. 
Proof. Using the constraint (16) we have

$$
\Sigma_{+}^{\prime}=(q-2)\left(\Sigma_{+}+1\right)+\frac{9}{2}\left(N_{1}^{2}-N_{1}\left(N_{2}+N_{3}\right)\right) .
$$

The constraint also yields $q-2 \leq 3 N_{1}\left(N_{2}+N_{3}\right)$ so that, if $-1<\Sigma_{+}<1 / 3$,

$$
\Sigma_{+}^{\prime}<\frac{1}{2}\left(9 N_{1}-N_{2}-N_{3}\right) N_{1}
$$

The lemma follows.

Lemma 17. Consider a Bianchi VIII solution to (14)-(10) and let $I=[t, s]$, where $s \leq 0$ and 0 belongs to the existence interval. There is an $\epsilon_{0}>0$ with the following property: for all $\epsilon_{0}>\epsilon>0$ there is an $A>0$ such that if $N_{2}(\tau), N_{3}(\tau) \geq A$ for all $\tau \in I$, then $-1+2 \epsilon \leq \Sigma_{+}(s)$ implies $-1+\epsilon \leq \Sigma_{+}(\tau)$ for all $\tau \in I$. A only depends on $\epsilon$ and the solution.

Proof. Let $\epsilon>0$. We have $\left|N_{1} N_{2} N_{3}\right| \leq C$ in $I$ where $C$ only depends on the solution. Consider

$$
\Sigma_{+}^{\prime}=(q-2)\left(\Sigma_{+}+1\right)+\frac{9}{2}\left(N_{1}^{2}-N_{1}\left(N_{3}+N_{3}\right)\right) .
$$

Observe that the last term is the only one pushing $\Sigma_{+}$in the negative direction, since $q \leq 2$ and $N_{1}<0$ for Bianchi VIII. In order for $\Sigma_{+}$to attain $-1+\epsilon$ in $I$ it must go between $-1+2 \epsilon$ and $-1+\epsilon$ in $I$. Due to (14) the last term of the right hand side of (33) will then behave as $\exp \left(2 \tau-2 \tau_{1}\right) \eta$, if $\epsilon$ is small enough, where $\tau_{1}$ corresponds to $-1+2 \epsilon$ and $\eta$ may be chosen arbitrarily small by choosing $A$ large enough. In consequence it is impossible for $\Sigma_{+}$to attain $-1+\epsilon$ if we choose $A$ big enough depending only on $\epsilon$ and the solution.

Proposition 2. A Bianchi IX solution has an $\alpha$-limit point.

Proof. Assume $\|N\| \rightarrow \infty$. By Lemma 15 we may assume $N_{1}, N_{1}\left(N_{2}+N_{3}\right) \rightarrow 0$ and $N_{2}, N_{3} \rightarrow \infty$ as $\tau \rightarrow-\infty$. Since $N_{2} N_{3} \rightarrow \infty$ we conclude that

$$
\int_{0}^{\tau}\left(\Sigma_{+}^{2}+\Sigma_{-}^{2}+\Sigma_{+}\right) d s \rightarrow \infty
$$

but then

$$
\int_{\tau}^{0} \Sigma_{+} d s \rightarrow-\infty
$$

Since $9 N_{1}<N_{2}+N_{3}$ for $\tau \leq T$ Lemma 16 and (35) yield the conclusion that $\Sigma_{+}<0 \forall \tau \leq T$.

Assume there is a $\tau_{2} \leq T$ such that $-1<\Sigma_{+}\left(\tau_{2}\right)$. Then $\Sigma_{+}\left(\tau_{2}\right) \leq \Sigma_{+}(\tau) \forall \tau \leq \tau_{2}$ by Lemma 16. Let $\mathcal{A}=\left\{\tau: \Sigma_{+}^{2}+\Sigma_{-}^{2}<-\Sigma_{+}\right\}$and $\mathcal{A}_{\tau}=\mathcal{A} \cap\left[\tau, \tau_{2}\right]$. Since $\Sigma_{+}^{\prime}<0 \forall \tau \leq \tau_{2}$, cf. the proof of Lemma 16, we have by (32)

$$
\begin{aligned}
\Sigma_{+}\left(\tau_{2}\right)-\Sigma_{+}(\tau) \leq & \int_{\mathcal{A}_{\tau}} \Sigma_{+}^{\prime} d s \leq\left(1+\Sigma_{+}\left(\tau_{2}\right)\right) \int_{\mathcal{A}_{\tau}}(q-2) d s \\
& \leq 2\left(1+\Sigma_{+}\left(\tau_{2}\right)\right) \int_{\mathcal{A}_{\tau}}\left(\Sigma_{+}^{2}+\Sigma_{-}^{2}+\Sigma_{+}\right) d s .
\end{aligned}
$$

By (34) the expression on the right tends to $-\infty$ as $\tau \rightarrow-\infty$ so that $\Sigma_{+}(\tau) \rightarrow \infty$ which is a contradiction. 
Assume there is an $S$ such that $\Sigma_{+}(\tau) \leq-1 \forall \tau \leq S$. Then the constraint yields $\left(\Sigma_{+}, \Sigma_{-}\right) \rightarrow(-1,0)$ and Proposition 1 yields $\Sigma_{-}=0$, so that all the $N_{i}$ must decrease for $\tau \leq S$ as we go backward in time by (14) and (15). We have a contradiction to our assumption.

In other words there is a sequence $\tau_{k}$ such that $N_{i}\left(\tau_{k}\right)$ is bounded. Since $q\left(\tau_{k}\right)$ is contained in a compact set by Lemma 6 we may extract a convergent subsequence and the proposition follows.

Corollary 3. Consider a Bianchi IX solution. For all $\epsilon>0$ there is a $T$ such that $\tau \leq T$ implies

$$
q(\tau) \leq 2+\epsilon
$$

Remark. For a Taub-NUT solution there is a $T$ such that $q(\tau)>2$ for all $\tau \leq T$.

Proof. Since there is an $\alpha$-limit point, Lemma 13 proves that there is a sequence $\tau_{k}$ such that $\left(N_{1} N_{2} N_{3}\right)\left(\tau_{k}\right) \rightarrow 0$ but since $N_{1} N_{2} N_{3}$ is monotone we may conclude that the product converges to zero. Let $10^{-3}>\eta>0$. Let $T$ be such that $\left(N_{1} N_{2} N_{3}\right)(\tau) \leq \eta^{3}$ for all $\tau \leq T$ and assume for a $\tau \leq T$ that $N_{3} \geq N_{2} \geq N_{1}$. We get $N_{1}(\tau) \leq \eta$. If, in $\tau, N_{3} \geq 2$ Lemma 6 yields $N_{1}\left(N_{2}+N_{3}\right) \leq \eta$. If $N_{3} \leq 2$ in $\tau$ we get $N_{1}\left(N_{2}+N_{3}\right) \leq 4 \eta$. Thus we have

$$
q \leq 2+12 \eta
$$

and the statement follows by the constraint (16).

Proposition 3. A Bianchi VIII solution has an $\alpha$-limit point.

Proof. Assume $\|N\| \rightarrow \infty$ as $\tau \rightarrow-\infty$. The consequences given in Lemma 15 will be used freely below. As $N_{2} N_{3} \rightarrow \infty$ we get

$$
\int_{\tau}^{0}\left(\Sigma_{+}^{2}+\Sigma_{-}^{2}+\Sigma_{+}\right) d s \rightarrow-\infty
$$

by inspecting (14). If $\Sigma_{+} \rightarrow-1$ Proposition 1 yields $N_{2}=N_{3}$ and $\Sigma_{-}=0$. By the constraint and Lemma $1+\Sigma_{+}$decays exponentially so that all the $N_{i}$ converge to finite values, contradicting our assumption. If not there is an $\epsilon>0$ and by Lemma 17 a $T$ such that $\tau \leq T$ implies $-1+\epsilon / 2 \leq \Sigma_{+}(\tau)$. We get, sooner or later,

$$
\begin{aligned}
\Sigma_{+}^{\prime}=(q-2)\left(\Sigma_{+}+1\right)+\frac{9}{2}\left(N_{1}^{2}-\right. & \left.N_{1}\left(N_{2}+N_{3}\right)\right) \leq\left(\Sigma_{+}^{2}+\Sigma_{-}^{2}+\Sigma_{+}-\Sigma_{+}-1\right) \epsilon \\
+ & \frac{9}{2}\left(N_{1}^{2}-N_{1}\left(N_{2}+N_{3}\right)\right) \leq\left(\Sigma_{+}^{2}+\Sigma_{-}^{2}+\Sigma_{+}\right) \epsilon
\end{aligned}
$$

so that

$$
\Sigma_{+}\left(\tau_{2}\right)-\Sigma_{+}(\tau) \leq \epsilon \int_{\tau}^{\tau_{2}}\left(\Sigma_{+}^{2}+\Sigma_{-}^{2}+\Sigma_{+}\right) d s \rightarrow-\infty
$$

by (36). But that is not possible. The existence of an $\alpha$-limit point follows as in the previous proposition. $\square$

Theorem 3. If a Bianchi VIII or IX solution to (14)-(10) is not contained in the invariant set $\Sigma_{-}=0, N_{2}=N_{3}$ or one of the sets found by applying the symmetries to it, the solution has an $\alpha$-limit point $\left(n_{1}, n_{2}, n_{3}, s_{+}, s_{-}\right)$for which $\left(s_{+}, s_{-}\right)$is not special. 
Proof. Consider a solution not contained in the invariant sets mentioned and let $\left(n_{1}, n_{2}, n_{3}, s_{+}, s_{-}\right)$be an $\alpha$-limit point. If $\left(s_{+}, s_{-}\right)$is not special we are done, so assume $\left(s_{+}, s_{-}\right)=(-1,0)$. Let $\tau_{k}$ be a sequence such that the solution evaluated at $\tau_{k}$ converges to the given $\alpha$-limit point. Since $\left(\Sigma_{+}, \Sigma_{-}\right)$does not converge to the special point there is an $0<\epsilon<10^{-3}$ such that for $k$ large enough we may for each $\tau_{k}$ find the first time, call it $s_{k} \leq \tau_{k}$, that $\left(\Sigma_{+}\left(s_{k}\right), \Sigma_{-}\left(s_{k}\right)\right) \in \partial B_{\epsilon}(-1,0)$. If there is a subsequence of $s_{k}$ such that $N_{i}\left(s_{k}\right)$ is bounded we are done so assume not.

The goal is to go backward in time starting at $s_{k}$ in order to produce a sequence with the desired properties. Since $q$ is bounded as we go backward in time we must have $\tau_{k}-s_{k} \rightarrow \infty$ so that $N_{1}\left(s_{k}\right) \rightarrow 0$ since $N_{1}^{\prime}=\left(q-4 \Sigma_{+}\right) N_{1}$ and $q-4 \Sigma_{+}$is roughly 6 in $B_{\epsilon}(-1,0)$. We get $N_{3}\left(s_{k}\right), N_{2}\left(s_{k}\right) \rightarrow \infty$ and $N_{1}\left(N_{2}+N_{3}\right) \rightarrow 0$ in $s_{k}$.

Bianchi $I X$. There is an $\eta>0$ such that $-1+\eta \leq \Sigma_{+}\left(s_{k}\right) \leq 0$ by the constraint if $k$ great enough, and then we also have $9 N_{1}\left(s_{k}\right)<N_{2}\left(s_{k}\right)+N_{3}\left(s_{k}\right)$. If the latter inequality holds $\forall \tau \leq s_{k}$ we get a contradiction to the existence of our limit point by Lemma 16. Let $v_{k} \leq s_{k}$ be the first time $9 N_{1}=N_{2}+N_{3}$ or one of $N_{2}, N_{3}$ becomes equal to $N_{1}$. Since $N_{1} N_{2} N_{3} \rightarrow 0$, cf. the proof of Corollary 3, we may assume

$$
N_{1} N_{2} N_{3} \leq 1
$$

If $N_{1}\left(v_{k}\right)=N_{2}\left(v_{k}\right) \leq N_{3}\left(v_{k}\right)$ then $N_{1}\left(v_{k}\right)=N_{2}\left(v_{k}\right) \leq 1$ by (37) and $N_{3}\left(v_{k}\right) \leq 10$ by Lemma 6 and similarly if $N_{2}$ and $N_{3}$ change roles. If $N_{1}\left(v_{k}\right)<N_{i}\left(v_{k}\right) i=2,3$ and $9 N_{1}\left(v_{k}\right)=N_{2}\left(v_{k}\right)+N_{3}\left(v_{k}\right)$, then $N_{1}\left(v_{k}\right) \leq 1$ and $N_{i}\left(v_{k}\right) \leq 9, i=2,3$. Regardless, we have $N_{i}\left(v_{k}\right) \leq 10$. For $k$ great enough there is a $t_{k}$ and a $u_{k}$ with $v_{k} \leq u_{k} \leq t_{k} \leq s_{k}$ such that $N_{3}\left(t_{k}\right)=10^{20}, N_{3}\left(u_{k}\right)=10^{10}$ and

$$
10^{10} \leq N_{3}(\tau) \leq 10^{20} \forall \tau \in\left[u_{k}, t_{k}\right] .
$$

In consequence

$$
10^{9} \leq N_{2}(\tau) \leq 10^{21} \quad \forall \tau \in\left[u_{k}, t_{k}\right]
$$

by Lemma 6. The inequalities above are used to prove that $t_{k}-u_{k} \geq 1$ from which we deduce the existence of an $r_{k} \in\left[u_{k}, t_{k}\right]$ such that $\left|\Sigma_{-}\left(r_{k}\right)\right| \leq 1 / 10$. That will suffice to prove the lemma.

We get

$$
20 \leq \ln \frac{N_{3}\left(t_{k}\right)}{N_{3}\left(u_{k}\right)}=\int_{u_{k}}^{t_{k}}\left(q+2 \Sigma_{+}-2 \sqrt{3} \Sigma_{-}\right) d s .
$$

But $N_{1}\left(N_{2}+N_{3}\right) \leq 2 \cdot 10^{-9}$ in $\left[u_{k}, t_{k}\right]$ by (37)-(39) so that $q \leq 3$ in that interval by the constraint (16), and thus $t_{k}-u_{k} \geq 1$. We have $\left|N_{2} / N_{3}-1\right| \leq 2 \cdot 10^{-10}$ in $\left[u_{k}, t_{k}\right]$ by the constraint and the above. If $\left|\Sigma_{-}\right| \geq 1 / 10$ in $\left[u_{k}, t_{k}\right]$ we get

$$
\left|\int_{u_{k}}^{t_{k}} 4 \sqrt{3} \Sigma_{-} d \tau\right| \geq \frac{3}{5}
$$

But

$$
\frac{N_{2}\left(t_{k}\right)}{N_{3}\left(t_{k}\right)}=\exp \left(\int_{u_{k}}^{t_{k}} 4 \sqrt{3} \Sigma_{-} d \tau\right) \frac{N_{2}\left(u_{k}\right)}{N_{3}\left(u_{k}\right)}
$$

and we have a contradiction. Consequently there is an $r_{k} \in\left[u_{k}, t_{k}\right]$ such that $-1+\eta \leq \Sigma_{+}\left(r_{k}\right),\left|\Sigma_{-}\left(r_{k}\right)\right| \leq 1 / 10$ and $N_{i}\left(r_{k}\right) \leq 10^{21}$. The conclusion of the theorem follows. 
Bianchi VIII. There is an $\eta>0$ such that $-1+2 \eta \leq \Sigma_{+}\left(s_{k}\right) \leq 0$. Let $A=A(\eta) \geq 1$ be as in Lemma 17 and $10 A \leq N_{3}(\tau) \forall \tau \in\left[v_{k}, s_{k}\right]$ and $N_{3}\left(v_{k}\right)=10 A$, observe that by the existence of the limit point the $N_{i}$ must become small. Then we may argue as in the Bianchi IX case.

\section{Conclusions}

Theorem 4. Consider a Bianchi VIII or IX solution of (14)-(10) which is not of NUT or Taub-NUT type respectively. Then there is a sequence $\tau_{k} \rightarrow-\infty$ such that $\tilde{\kappa}\left(\tau_{k}\right) \rightarrow c \neq 0$.

Proof. The Taub-NUT and the NUT solutions correspond to $\Sigma_{-}=0$ and $N_{2}=N_{3}$ or one of the sets obtained by applying the symmetries. By Theorem 3 and Lemma 14 the theorem follows.

Theorem 5. For a Bianchi VIII or IX solution to (14)-(10) which is not a NUT or Taub-NUT solution $\left(\Sigma_{+}, \Sigma_{-}\right)$cannot converge to a special point on the Kasner circle and the $\alpha$-limit set contains at least two distinct points of type I, at least one of which is non-flat.

Proof. The first statement is Proposition 11 and the last statement follows from Theorem 3 and Lemma 14.

Observe that this theorem says that the solution does not converge; the shear variables will oscillate indefinitely.

Proof of Theorem [. Let $(M, g)$ be the globally hyperbolic Lorentz manifold obtained in Lemma 2. Assume there is a connected Lorentz manifold $(\hat{M}, \hat{g})$ of the same dimension and a map $i: M \rightarrow \hat{M}$ which is an isometry onto its image with $i(M) \neq \hat{M}$. Then there is a $p \in \hat{M}-i(M)$ and a timelike geodesic $\gamma:[a, b] \rightarrow \hat{M}$ such that $\gamma([a, b)) \subseteq i(M)$ and $\gamma(b)=p$. Since $\left.\gamma\right|_{[a, b)}$ can be considered to be a future or past inextendible timelike geodesic in $M$ either it has infinite length or the Kretschmann scalar blows up along it, combining Lemma 2, 3 and Theorem 4. Both possibilities lead to a contradiction. Observe that this proves that $(M, g)$ is the maximal globally hyperbolic development. That the Kretschmann scalar is unbounded in the incomplete directions of inextendible causal geodesics also follows from Lemma 2,3 and Theorem 4 .

\section{APPENDIX}

We here prove the technical results of Lemma 2 and 3 .

Proof of Lemma . We begin by proving that we obtain a solution to Einstein's vacuum equation with the correct initial conditions. Let $e_{i}^{\prime}, i=1,2,3$ be a left invariant orthonormal basis. We can assume the corresponding $n^{\prime}$ to be of one of the forms given in table 1 by Lemma 1. The content of (欧) is that $k_{i j}=k\left(e_{i}^{\prime}, e_{j}^{\prime}\right)$ and $n^{\prime}$ are to commute. We may thus also assume $k_{i j}$ to be diagonal without changing the earlier conditions of the construction. If we let $n\left(t_{0}\right)=n^{\prime}, \theta\left(t_{0}\right)=\operatorname{tr}_{g} k$ and $\sigma_{i j}\left(t_{0}\right)=k_{i j}-\theta \delta_{i j} / 3$ then (3) is the same as (12). Let $n, \sigma$ and $\theta$ satisfy (8), (10) and (11) with initial values as specified above. Since (12) is satisfied at $t_{0}$ it is satisfied for all times. For reasons given in connection with 12 and $\sigma$ will remain diagonal so that (9) will always hold. 
Let $M=I \times G$ where $I$ is the maximal existence interval for solutions to (8)(12). We construct a basis $e_{\alpha}$, define a metric by demanding that the basis be orthonormal and show that the corresponding $\tilde{n}, \tilde{\sigma}$ and $\tilde{\theta}$ coincide with $n, \sigma$ and $\theta$. We will thereby have constructed a Lorentz manifold satisfying Einstein's vacuum equations with the correct initial conditions.

Let $n_{i}$ and $\sigma_{i}$ denote the diagonal elements of $n$ and $\sigma$ respectively. Let $f_{i}\left(t_{0}\right)=1$ and $\dot{f}_{i} / f_{i}=2 \sigma_{i}-\theta / 3$. Let $a_{1}=\left(f_{2} f_{3}\right)^{1 / 2}, a_{2}=\left(f_{1} f_{3}\right)^{1 / 2}, a_{3}=\left(f_{1} f_{2}\right)^{1 / 2}$ and define $e_{i}=a_{i} e_{i}^{\prime}$. Then $\tilde{n}$ associated to $e_{i}$ equals $n$. We complete the basis by letting $e_{0}=\partial_{t}$. Define a metric $<\cdot, \cdot>$ on $M$ by demanding $e_{\alpha}$ to be orthonormal with $\left\langle e_{0}, e_{0}\right\rangle=-1$ and $\left\langle e_{i}, e_{i}\right\rangle=1, i=1,2,3$ and let $\nabla$ be the associated Levi-Civita connection. Compute $<\nabla_{e_{0}} e_{i}, e_{j}>=0$. If $\tilde{\theta}(X, Y)=<\nabla_{X} e_{0}, Y>$ and $\tilde{\theta}_{\mu \nu}=\tilde{\theta}\left(e_{\mu}, e_{\nu}\right)$, then $\tilde{\theta}_{00}=\tilde{\theta}_{i 0}=\tilde{\theta}_{0 i}=0$. Furthermore,

$$
\frac{1}{a_{j}} e_{0}\left(a_{j}\right) \delta_{i j}=-\tilde{\theta}_{i j}
$$

(no summation over $j$ ) so that $\tilde{\theta}_{i j}$ is diagonal and $\operatorname{tr} \tilde{\theta}=\theta$. Finally,

$$
-\tilde{\sigma}_{i i}=-\tilde{\theta}_{i i}+\frac{1}{3} \theta=-\sigma_{i} .
$$

The constructed Lorentz manifold thus satisfies Einstein's vacuum equations. Next we prove that each $M_{v}=\{v\} \times G$ is a Cauchy surface. The metric is given by

$$
-d t^{2}+\sum_{i=1}^{3} a_{i}^{-2}(t) \xi^{i} \otimes \xi^{i}
$$

where $\xi^{i}$ are the duals of $e_{i}^{\prime}$. A causal curve cannot intersect $M_{v}$ twice since the $t$ component of such a curve must be strictly monotone. Assume that $\gamma:\left(s_{-}, s_{+}\right) \rightarrow$ $M$ is an inextendible causal curve that never intersects $M_{v}$. Let $t: M \rightarrow I$ be defined by $t[(s, h)]=s$. Let $s_{0} \in\left(s_{-}, s_{+}\right)$and assume that $t\left(\gamma\left(s_{0}\right)\right)=t_{1}<v$ and that $<\gamma^{\prime}, \partial_{t}><0$ where it is defined. Thus $t(\gamma(s))$ increases with $s$ and $t\left(\gamma\left(\left[s_{0}, s_{+}\right)\right)\right) \subseteq\left[t_{1}, v\right]$. Since we have uniform bounds on $a_{i}$ from below and above on $\left[t_{1}, v\right]$ and the curve is causal we get

$$
\left(\sum_{i=1}^{3} \xi^{i}\left(\gamma^{\prime}\right)^{2}\right)^{1 / 2} \leq-C<\gamma^{\prime}, e_{0}>
$$

on that interval, with $C>0$. Since

$$
\int_{s_{0}}^{s_{+}}-<\gamma^{\prime}, e_{0}>d s=\int_{s_{0}}^{s_{+}} \frac{d t \circ \gamma}{d s} d s \leq v-t_{1}
$$

the curve $\left.\gamma\right|_{\left[s_{0}, s_{+}\right)}$, projected to $G$, will have finite length in the metric $\rho$ on $G$ defined by making $e_{i}^{\prime}$ an orthonormal basis. Since $\rho$ is a left invariant metric on a Lie group it is complete and sets closed and bounded in the corresponding topological metric must be compact. Adding the above observations, we conclude that $\gamma\left(\left[s_{0}, s_{+}\right)\right)$is contained in a compact set. For each sequence $s_{k} \rightarrow s_{+}$- there is thus a subsequence $s_{n_{k}}$ such that $\gamma\left(s_{n_{k}}\right)$ converges. Since $t(\gamma(s))$ is monotone it converges. We cannot have two limit points since that would contradict the causality of $\gamma$. Thus $\gamma$ must converge as $s \rightarrow s_{+}$so that it is extendible. By this and similar arguments covering the other cases, we conclude that $M_{v}$ is a Cauchy surface for each $v \in\left(t_{-}, t_{+}\right)$. 
Next we prove the statements made in Lemma 2 concerning causal geodesic completeness. Let us first time orient the different manifolds. Consider manifolds which are not of type IX. Then the constraint (12) yields the inequality $\sigma_{i j} \sigma^{i j} \leq 2 \theta^{2} / 3$, cf. (27). Then the Raychaudhuri equation yields $\left|e_{0}(\theta)\right| \leq \theta^{2}$. Consequently, if $\theta$ is once zero it is always zero. By considering equations (8)-(12) one concludes that $\theta=0$ is only possible if $\sigma_{i j}=n_{i j}=0$ or if two of the diagonal elements of $n_{i j}$ are equal and constant, the third is zero and $\sigma_{i j}=0$. Time orient the manifolds which are not of type IX and which do not have $\theta=0$ by demanding that $\theta$ be positive.

Observe that for a manifold which is not of type IX, $\theta$ decreases in magnitude with time, so that it is bounded to the future. By the constraint (12), the same is true of $\sigma_{i j}$. Using (8) we get control of $n_{i j}$ and conclude that the solution may not blow up in finite time. The interval $I=\left(t_{-}, t_{+}\right)$in Lemma 2 must thus have $t_{+}=\infty$.

We now prove future causal geodesic completeness for manifolds which are not of type IX. Let $\gamma:\left(s_{-}, s_{+}\right) \rightarrow M$ be a future directed inextendible causal geodesic. We prove that $s_{+}$must be infinite. Let the function $t$ be defined as in the previous lemma. Since every $M_{v}, v \in I$ is a Cauchy surface, $t(\gamma(s))$ must cover the interval $I$ as $s$ runs through $\left(s_{-}, s_{+}\right)$. Furthermore, $t(\gamma(s))$ is monotone increasing. Define

$$
f_{\mu}(s)=<\gamma^{\prime}(s),\left.e_{\mu}\right|_{\gamma(s)}>.
$$

Let $s_{0} \in\left(s_{-}, s_{+}\right)$and compute

$$
\int_{s_{0}}^{s}-f_{0}(u) d u=t(\gamma(s))-t\left(\gamma\left(s_{0}\right)\right)
$$

so that the right hand side goes to $\infty$ as $s \rightarrow s_{+}$. If we can prove that $f_{0}$ may not become unbounded in a finite $s$-interval, we are done. If $\theta \equiv 0$, then $\left(t_{-}, t_{+}\right)=(-\infty, \infty)$ and $f_{0}$ is constant so that all causal geodesics are future and past complete. We exclude this case from now on. Compute

$$
\frac{d f_{0}}{d s}=<\gamma^{\prime}(s), \nabla_{\gamma^{\prime}(s)} e_{0}>=\sum_{k=1}^{3} \theta_{k} f_{k}^{2}
$$

where $\theta_{k}$ are the diagonal elements of $\theta_{i j}$. Consider functions of $t$ as functions of $s$ by evaluating them at $t(\gamma(s))$. Compute, using Raychaudhuri's equation (11),

$$
\frac{d}{d s}\left(f_{0} \theta\right)=\frac{1}{3} \theta^{2} \sum_{k=1}^{3} f_{k}^{2}+\sum_{k=1}^{3} \theta \sigma_{k} f_{k}^{2}+f_{0}^{2} \sum_{k=1}^{3} \sigma_{k}^{2}+\frac{1}{3} \theta^{2} f_{0}^{2}
$$

where $\sigma_{k}$ are the diagonal elements of $\sigma_{i j}$. Estimate

$$
\left|\sum_{k=1}^{3} \sigma_{k} f_{k}^{2}\right| \leq\left(\frac{2}{3}\right)^{1 / 2}\left(\sum_{k=1}^{3} \sigma_{k}^{2}\right)^{1 / 2} \sum_{k=1}^{3} f_{k}^{2}
$$

using the tracelessness of $\sigma_{i j}$. By making a division into the three cases $\sum_{k=1}^{3} \sigma_{k}^{2} \leq$ $\theta^{2} / 3, \theta^{2} / 3 \leq \sum_{k=1}^{3} \sigma_{k}^{2} \leq 2 \theta^{2} / 3$ and $2 \theta^{2} / 3 \leq \sum_{k=1}^{3} \sigma_{k}^{2}$ and using the causality of $\gamma$ we deduce

$$
\frac{d}{d s}\left(f_{0} \theta\right) \geq \frac{2-\sqrt{2}}{3} \theta^{2} f_{0}^{2} .
$$

Observe that this estimate holds for a Bianchi IX solution as well. 
Since $f_{0} \theta$ is negative on $\left[s_{0}, s_{+}\right)$, its absolute value is thus bounded on that interval. If $s_{+}$were finite, $\theta$ would be bounded from below by a positive constant on $\left[s_{0}, s_{+}\right)$, since

$$
\left|\frac{d \theta}{d s}\right| \leq-f_{0} \theta^{2} \leq C \theta
$$

on that interval for some $C>0$. Then (41) would imply the boundedness of $f_{0}$ on $\left[s_{0}, s_{+}\right)$. That would contradict (40).

Excluding Bianchi IX for the moment, the inequality (41) also proves that all causal geodesics are past incomplete since it implies that $f_{0} \theta$ must blow up after a finite $s$-time going into the past.

Consider a Bianchi IX manifold. By a theorem by Lin and Wald (1989a), the trace of the second fundamental form will be zero for some $t_{0} \in I=\left(t_{-}, t_{+}\right.$) (in their paper they demand that $G$ have topology $S^{3}$, but this is not necessary for their argument). However, on $I_{-}=\left(t_{-}, t_{0}\right)$ and on $I_{+}=\left(t_{0}, t_{+}\right) \theta \neq 0$ since it is only zero once (this can be seen by inspecting equations (8)-(12)). A future directed inextendible causal geodesic $\gamma$ in a Bianchi IX manifold must consequently have points $s_{1}, s_{2}$ with $\theta(t(\gamma(s)))$ positive (negative) for $s \leq s_{1}\left(s \geq s_{2}\right)$. The inequality (41) now implies that the geodesic cannot be defined more than a finite interval before $s_{1}$ nor more than a finite interval after $s_{2}$. We have future and past causal geodesic incompleteness. We also conclude that $t_{-}>-\infty$ for all manifolds that do not satisfy $\theta \equiv 0$ and $t_{+}<\infty$ for Bianchi IX manifolds since the curve defined by $\gamma(s)=(s, e)$ is a geodesic.

Finally, we prove the last statement of Lemma 2. Assume the Kretschmann scalar is unbounded as $t \rightarrow t_{-}$and let $\gamma$ be a past inextendible causal geodesic. Since each $M_{v}$ is a Cauchy surface, $\gamma$ must pass through each of them and thus the Kretschmann scalar must be unbounded along it.

Proof of Lemma 9. Consider a Bianchi IX solution of (8)-(12). As observed in the proof of Lemma 2 the existence interval $I=\left(t_{-}, t_{+}\right)$can be divided into $I_{-}=$ $\left(t_{-}, t_{0}\right), I_{+}=\left(t_{0}, t_{+}\right)$and $t_{0}$, where $t_{0}$ is the only zero of $\theta$ in $I$. We now relate the different time coordinates on $I_{-}$.

According to equation (13) $\tau$ has to satisfy $d t / d \tau=3 / \theta$. Define $\tau(t)=\int_{t_{1}}^{t} \theta(s) / 3 d s$, where $t_{1} \in I_{-}$. Then $\tau: I_{-} \rightarrow \tau\left(I_{-}\right)$is a diffeomorphism and strictly monotone on $I_{-}$. By equation (11) $\theta$ decreases so that it will be positive in $I_{-}$and $\tau$ will increase with $t$.

Since $\theta$ is continuous beyond $t_{0}$ it is clear that $\tau(t) \rightarrow \tau_{0} \in \mathbb{R}$ as $t \rightarrow t_{0}$. To prove that $t \rightarrow t_{-}$corresponds to $\tau \rightarrow-\infty$ we make the following observation. One of the expressions $\theta$ and $d \theta / d t$ is unbounded on $\left(t_{-}, t_{1}\right]$, since if both were bounded the same would be true of $\sigma_{i j}$ and $n_{i j}$ by (11) and (8) respectively. Then we would be able to extend the solution beyond $t_{-}$, contradicting the fact that $I$ is the maximal existence interval (observe that $t_{-}>-\infty$ by the proof of Lemma 2). If $\tau$ were bounded from below on $I_{-}$, then $\theta$ and $\theta^{\prime}$ would be bounded on $\tau\left(\left(t_{-}, t_{1}\right]\right)$ by Lemma 5, and thus $\theta$ and $d \theta / d t$ would be bounded on $\left(t_{-}, t_{1}\right]$. Thus $t \rightarrow t_{-}$ corresponds to $\tau \rightarrow-\infty$. Since $\theta \rightarrow \infty$ as $\tau \rightarrow-\infty$ by (18), $\kappa\left(\tau_{k}\right)=\tilde{\kappa}\left(\tau_{k}\right) \theta^{4}\left(\tau_{k}\right)$ is unbounded. Consequently

$$
\limsup _{t \rightarrow t_{-}}\left|R_{\alpha \beta \gamma \delta} R^{\alpha \beta \gamma \delta}\right|=\infty
$$


for all non-Taub-NUT initial data given the assumptions of the Lemma. Similar arguments yield the same conclusion for $t \rightarrow t_{+}$and for $t \rightarrow t_{-}$given non-NUT Bianchi VIII initial data.

\section{ACKNOWLEDGMENTS}

The author would like to express his gratitude to his advisor, Lars Andersson, for suggesting the problem and helpful discussions, and to Alan Rendall for reading the argument and suggesting improvements.

\section{REFERENCES}

Berger B K. Garfinkle D, Isenberg J, Moncrief V and Weaver M 1998 Mod. Phys. Lett. A13 1565-74, gr-qc/9805063

Chruściel P and Rendall A 1995 Ann. Phys. 242 349-85, gr-qc/9410040

Ellis G and MacCallum M 1969 Commun. Math. Phys. 12 108-41

Hobill D, Burd A and Coley A 1994 Deterministic Chaos in General Relativity (New York: Plenum)

Lin X-F and Wald R 1989a Phys. Rev. D 40 3280-86

Lin X-F and Wald R 1989b Phys. Rev. D $412444-48$

Rendall A 1997 Class. Quantum Grav. 14 2341-56, gr-qc/9703036

Wainwright J and Hsu L 1989 Class. Quantum Grav. 6 1409-31

Wainwright J and Ellis G F R editors 1997 Dynamical Systems in Cosmology (Cambridge:

Cambridge University Press) 40

Department of Mathematics, Royal Institute of Technology, S-100 44 Stockholm, SweDEN 